\title{
Reconsidering Research on Learning from Media
}

\author{
Richard E. Clark \\ University of Southern California
}

\begin{abstract}
Recent meta-analyses and other studies of media's influence on learning are reviewed, Consistent evidence is found for the generalization that there are no learning benefits to be gained from employing any specific medium to deliver instruction. Research showing performance or timesaving gains from one or another medium are shown to be vulnerable to compelling rival hypotheses concerning the uncontrolled effects of instructional method and novelty. Problems with current media attribute and symbol system theories are described and suggestions made for more promising research directions.
\end{abstract}

Studies of the influence of media on learning have ken a fixed feature of educational research since Thorndike (1912) recommended pictures as a laborsaving device in instruction, Most of this research is buttressed by the hope that learning will be enhanced with the proper mix of medium, student, subject matter content, and learning task. A typical study compares the relative achievement of groups who have received similar subject matter from different media. This research has led to so-called 'media selection" schemes or models 1982). These models generally promise to incorporate existing research and practice into procedures for selecting the best medium or mix of media to deliver instruction.

Most of these models base many of their prescriptions on presumed learning benefits from media (Jamison, Suppes, \& Welles, 1974).

However, this article will argue that most current summaries and meta-analyses of media comparison studies clearly suggest that media do not influence learning under any conditions. Even in the few cases where dramatic changes in achievement or ability have followed the introduction of a medium, as was the case with television in El Salvador (Schramm, 1977), it was not the medium that caused the change but rather a curricular reform that accompanied the change. The best current evidence is that media are mere vehicles that deliver instruction but do not influence student achievement any more than the truck that delivers our groceries causes changes in our nutrition. Basically, the choice of vehicle might influence the cost or extent of distributing instruction, but only the content of the vehicle can influence achievement. While research often shows a slight learning advantage for newer media over more conventional instructional vehicles, this advantage will be

The author wishes to acknowledge the substantive advice of Gavriel Salomon, William Winn, and anonymous reviewers without making them responsible for errors.

$$
\text { p } 445
$$

shown to be vulnerable to compelling rival hypotheses. Among these rival explanations is evidence of artifact and confounding in existing studies and biased editorial decisions which may favor research showing larger effect sizes for newer media. After summarizing evidence from current meta-analyses of media research, I will discuss advantages and problems with current "media attribute" and 'symbol system" theories and will conclude by suggesting tentative solutions to past problems and future directions for research involving media.

\section{Media Comparison Studies}

In the 1960s, Lumsdaine (1963) and others (e.g,, Mielke, 1968) argued that gross media comparison and selection studies might not pay off. They implied that media, when viewed as collections of mechanical instruments, such as television and computers, were sample delivery devices. Nevertheless, earlier reviewers also held the door open to learning effects from media by attributing much of the lack of significance in prior research to poor design and lack of adequate models or theory.

Lumsdaine (1963) dealt primarily with adequate studies that had used defensible methodology, and had found significant differences between treatments. With the benefit of hindsight it is not surprising that most of the studies he selected for review employed media as simple vehicles for instructional methods, such as text organization, size of step in programming, cueing, repeated exposures, and prompting. These studies compared the effects of. for 
example, different step size in programmed instruction via television. It was step size (and other methods), not television (or other media), which were the focus of thew studies. This is an example of what Salomon and Clark (1977) called research with media. In these studies media are mere conveyances for the treatments being examined and are not the focus of the study, though the results are often mistakenly interpreted as suggesting benefits for various media. An example of instructional research with media would be a study which contrasted a logically organized audiotutorial lesson on photosynthesis with a randomly sequenced presentation of the same frames (cf. Clark \& Snow, 1975; Salomon \& Clark, 1977, for a review of similar studies). Perhaps as a result of this confusion, Lumsdaine (1963) reached few conclusions beyond the suggestion that media might reduce the cost of instruction when many students are served because "the cost of perfecting it can be prorated in terms of a denominator representing thousands of students" (p. 670).

A decade later, Glaser and Cooley (1973) and Levie and Dickie (1973) were cautious about media comparison studies, which apparently were still being conducted in large numbers. Glaser and Cooley (1973) recommended using acceptable medium as "a vehicle for making available to schools what psychologists have learned about learning" (p. 855). Levie and Dickie (1973) noted that most media comparison studies to that date had been fruitless and suggested that objectives can be attained through "instruction presented by any of a variety of different media" (p. 859). At that time televised education was still a lively topic and studies of computerized instruction were just beginning to appear.

During the past decade, television research seems to have diminished considerably, but computer learning studies are now popular. This current $\mathrm{h}$ belongs to the familiar but generally fruitless media comparison approach or is concerned

$$
\text { p } 446
$$

with different contents or methods being presented via different media (e.g., science teaching via computers). Generally, each new medium seems to attract its own set of advocates who make claims for improved learning and stimulate research questions which are similar to those asked about the previously popular medium. Most of the radio research approaches suggested in the 1950s (e.g., Hovland, Lumsdaine, \& Sheffield, 1949) were very similar to those employed by the television movement of the 1960s (e.g., Schramm, 1977) and to the more recent reports of the computer-assisted instruction studies of the 1970s and 1980s (e.g., Dixon \& Judd, 1977). It seems that similar research questions have resulted in similar and ambiguous data. Media comparison studies, regardless of the media employed, tend to result i n "no significant difference" conclusions (Mielke, 1968). These findings were incorrectly offered-as-evidence that different media were "equally effective" as conventional means in promoting learning. No significant difference results simply suggest that changes in the outcome scores (e.g., learning) did not result from any systematic differences in the treatments compared.

Occasionally a study would find evidence for one or another medium. When this happens, Mielke (1968) has suggested that the active ingredient might be some uncontrolled aspect of the content or instructional strategy rather than the medium. When we investigate these positive studies, we find that the treatments are confounded. The evidence for this confounding may be found in the current meta-analyses of media comparison studies. The next section argues that it is the uncontrolled effects of novelty and instructional method which account for the existing evidence for the effects of various media on learning gains.

\section{Reviews and Meta-analyses of Media Research}

One of the most interesting trends in the past decade has been a significant increase in the number of excellent reviews and meta-analyses of research comparing the learning advantage of various media. The results of these overviews of past comparison studies seem to be reasonably unambiguous and unanimous. Taken together, they provide strong evidence that media comparison studies that find causal connections between media and achievement are confounded.

\section{Size of Effect of Media Treatments}

A recent series of meta-analyses of media comparison studies have been conducted by James Kulik and his colleagues at the University of Michigan (Cohen, Ebling \& Kulik, 1981; C. Kulik, Kulik, \& Cohen, 1980; J. Kulik, Bangert, \& Williams, 1983; J. Kulik, Kulik, \& Cohen, 1979). These reviews employ the relatively new technology of meta-analysis (Glass, 1976), which provides more precise estimates of the effect size of various media treatments than were possible a few years ago. Previous reviews dealing primarily with "box score" sums of significant 
findings for media versus conventional instructional delivery were sometimes misleading. Effect size estimates often were expressed in portions of standard score advantages for one or another type of treatment. This discussion will express effects in one of two ways: (a) the number of standard deviations separating experimental and control groups, and (b) as improvements in percent) scores on a final examination.

\section{p 447}

\section{Box Scores versus Effect Size}

An illustration of the advantage of meta-analytical effect size descriptions of past research over "box scores" is available in a recent review of Postlethwait's audiotutorial instruction studies (J. Kulik, Kulik, \& Cohen,, 1979). The authors found 42 adequate studies, of which 29 favored audiotutorial instruction and only 13 favored conventional instruction. Of those 42, only 15 reported significant differences, but 11 of the 15 favored audiotutorial and only 4 favored conventional instruction. This type of box score analysis would strongly favor the learning benefits of the audiotutorial approach over more conventional means, whereas effect size estimates of this data show only .2 standard deviations differences in the final exam scores of audiotutorial and conventional treatments. Kulik and his colleagues reported that this difference was equivalent to approximately 1.6 points on a 100-point final examination. This small effect is not instructionally significant and could easily be due to confounding.

The most common sources of confounding in media research seem to be the uncontrolled effects of (a) instructional method or content differences between treatments that are compared, and (b) a novelty effect for newer media, which tends to disappear over time.

\section{Uncontrolled Method and Content Effects}

In effect size analyses, all adequate studies are surveyed. They involve a great variety of subject matter content, learning task types, and grade levels. The most common result of this type of survey is a small and positive effect for newer media over more conventional instructional delivery devices. However, when studies are subjected to meta-analysis, our first source of rival hypotheses, medium and method confusion, shows up.

The positive effect for media more or less disappears when the same instructor produces all treatments (C. Kulik, Kulik, \& Cohen, 1980). Different teams of instructional designers or different teachers probably give different content and instructional methods to the treatments that are compared. If this is the case, we do not know whether to attribute the advantage to the medium or to the differences between content and method and the media being compared. However, if the effect for media tends to disappear when the same instructor or team designs contrasting treatments, we have reason to believe that the lack of difference is due to greater control of nonmedium variables. It was Mielke (1968) who reminded us that when examining the effects of different media, only the media being compared can be different. All other aspects of the treatments, including the subject matter content and method of instruction, must be identical.

\section{Meta-analytic Evidence for Method and Content Confounding}

In meta-analyses of college-level computerized versus conventional courses, an effect size of .51 results when different faculty teach the compared course (C. Kulik, Kulik, \& Cohen, 1980). This effect reduces to .13 when one instructor plans and teaches both experimental and control courses. Presumably, the weak but positive finding for college use' of computers over conventional media is due to systematic

\section{p 448}

but uncontrolled differences in content and/or method, contributed unintentionally

by different teachers or designers.

Time savings with computers. Another instance of this artifact may be found in studies that demonstrate considerable time savings due to certain media. Comparisons of computer and conventional instruction often show 30 to 50 percent reductions in time to complete lessons for the compute r groups (C. Kulik, Kulik, \& Cohen, 1980; Kulik, Bangert, \& Williams, 1983). A plausible rival hypothesis here is the possible effects of the greater effort invested in newer media programs than in conventional presentations of the same material. Comparing this increased effort invested in computer instruction to that afforded conventional instruction might be likened to sponsoring a race between a precision engineered racer and the family car. The difference in effort presumably involves more instructional design and development, which results in more effective instructional methods for the students in computer treatments. Presumably, the students in other treatments would fare as well if given the 
advantage of this additional design effort, which produces more effective presentations requiring less time to complete.

\section{Exchanging Method for Media in Instructional Research}

There is evidence in these meta-analyses that it is the method of instruction that leads more directly and powerfully to learning. Glaser (I 976) defines instructional methods as "the conditions which can be implemented to foster the acquisition of competence" (p. 1). It seems not to be media but variables such as instructional methods that foster learning. For example, instructional programs such as the Keller (I 968) personalized system of instruction (PSI) and programmed instruction (PI) contain methods which seek to add structure, shorter steps, reduced verbal loads, and self-pacing to lessons. Each, however, is typically associated with a different medium. The PSI (Keller plan) approach is usually presented by text, and PI is often the preferred approach of those who design computer-assisted instruction.

When studies of PI via text and via computer-assisted instruction are compared for their effect size they are similar. Both seem to show about a .2 standard deviation final examination advantage over conventional instruction (C. Kulik, Kulik, \& Cohen, 1980). A compelling hypothesis to explain this similarity might be that most computerized instruction is merely the presentation of PI or PSI via a computer.

When computer and PI effects are compared with the use of visuals in televised or audiotutorial laboratories, the PI and computer studies show about a 30 percent larger effect size. The largest effect size however, is reserved for the PSI approach. The description of this instructional program tends to focus on its essential methods rather than on a medium. Perhaps as a result, it typically results in a .5 standard deviation effect size when compared with conventional, computer, PI, or visual instruction (C. Kulik, Kulik, \& Cohen, 1980). This would indicate that when we begin to separate method from medium we may begin to explain more significant amounts of learning variance.

\section{Uncontrolled Novelty Effects with Newer Media}

A second, though probably less important source of confounding, is the increased effort and attention research subjects tend to give to media that are novel to them.

$$
\text { p } 449
$$

The increased attention paid by students sometimes results in increased effort or persistence, which yields achievement gains. If they are due to a novelty effect, these gains tend to diminish as students become more familiar with the new medium. This was the case in reviews of computer-assisted instruction at the secondary school level (grades 6 to 12) (Kulik, Bangert, \& Williams, 1983). An average effect size of .32 (e.g., a rise in exam scores from the 50th to the 63rd percentile) for computer courses tended to dissipate significantly in longer duration studies. In studies lasting 4 weeks or less, computer effects were .56 standard deviations. This reduced to .3 in studies lasting 5 to 8 weeks and further reduced to the familar.2 effect after 8 weeks of data collection. Cohen (1977) describes an effect size of .2 as "weak" and notes that it accounts for less than I percent of the variance in a comparison. Cohen, Ebling, and Kulik (1981) report a similar phenomenon in their review of visual-based instruction (e.g., film, television, pictures). Although the reduction in effect size for longer duration studies approached significance (about .065 alpha), there were a number of comparisons of methods mixed with different visual media, which makes interpretation difficult.

In their review of computer use in college, C. Kulik, Kulik, and Cohen (1980) did not find any evidence for this novelty effect. In their comparison of studies of I or 2 hours duration with those which held weekly sessions for an entire semester, the effect sizes were roughly the same. Computers are less novel experiences for college subjects than for secondary school students.

\section{Editorial Decisions and Distortion of Effect Estimates}

There is also some evidence for the hypothesis that journal editors typically select research that finds stronger effects for newer media. Kulik, Bangert, and Williams (1983) reported .21 and .3 effect sizes for unpublished and dissertation studies respectively. Published studies averaged effect sizes of .47 standard deviations, which is considerably larger. J. Kulik, Kulik, and Cohen (1979) found similar evidence in an analysis of audiotutorial instruction studies. Published studies showed a 3.8 percent final examination advantage for audiotutorial methods over conventional instruction (.31 standard deviations), but this reduced to a .6 percent advantage for the same method in unpublished studies. 


\section{A Research Caution}

Based on this consistent evidence, it seems reasonable to advise strongly against future media comparison research. Five decades of research suggest that there are no learning benefits to be gained from employing different media in instruction, regardless of their obviously attractive features or advertised superiority. All existing surveys of this research indicate that confounding has contributed to the studies attributing learning benefits to one medium over another and that the great majority of these comparison studies clearly indicate no significant differences.

This situation is analogous to the problems encountered in research on teaching. In that area, the teacher was constantly confused with teaching. Improvements in research findings result when specific teaching behaviors compete to influence learning rather than different types of teachers (Rosenshine, 1971). Where learning benefits are at issue, therefore, it is the method, aptitude, and task variables of

\section{p 450}

instruction that should be investigated. Studies comparing the relative achievement advantages of one medium over another will inevitably confound medium with method of instruction.

\section{Recent Directions: Media Attribute Research Examined}

During the 1970s a new type of question was suggested, which seemed to eliminate many of the conceptual problems in the media comparison question. Instead of focusing on media per se, it was recommended (Clark, 1975; Levie \& Dickie, 1973; Salomon, 1974b; 1979) that we study "attributes" of media and their influence on the way that information is processed in learning. In this view, many media possess attributes such as the capacity to slow the motion of objects or stimulus field or to "unwrap" a three-dimensional object into its two-dimensional form. These attributes were thought to cultivate cognitive skills when modeled by learners, so that, for example, a child with low cue attending ability might learn the cognitive skill of "zooming" into stimulus details (Salomon, 1974a), or novice chess players might increase their skills in recognizing potential moves and configurations of chess pieces through animated modeling of moves and patterns (Blake, 1977). Because this type of question dealt with the way that information is selected and transformed in the acquisition of generalizable cognitive skills, many believed that the possibility of a coherent theory dealing with media attributes was forthcoming (Olson, 1972; Schramm, 1977). In addition, it was exciting to imagine that these media attributes might result in unique cognitive skills because they promised to teach mental transformations which had not heretofore been experienced.

The promise of the media attributes approach is based on at least three expectations: (a) that the attributes were an integral part of media and would provide a connection between instructional uses of media and learning; (b) that attributes would provide for the cultivation of cognitive skills for learners who needed them; and (c) that identified attributes would provide unique independent variables for instructional theories that specified causal relationships between attribute modeling and learning-finally the evidence for a connection between media and learning. While the final point is most important, it now appears that the media attribute question has many of the problems that plagued the media comparison issue. Generally, the evidence suggests that only the second expectation has been fulfilled, which implies that media attribute research may contribute to instructional design but not to theory development.

\section{Media Attributes and Media}

The first expectation was that these media attributes would somehow represent the psychologically relevant aspects of media. Few of the originators of the media attribute construct (Olson \& Bruner, 1974; Salomon 1974b) claimed that they were more then "correlated" with different media. Since they were not exclusive to any specific media and were only associated with them by habit or convenience, they were not "media" variables any more than the specific subject matter content of a book is part of the definition of "book." In fact, the early discussions of the construct most often referred to "symbol systems" or symbolic "elements" of instruction. All instructional messages were coded in some symbolic representa-

$$
\text { p } 451
$$

tional system, the argument went, and symbols vary in the cognitive transformation they allow us to perform on the information we select from our environment. Some symbolic elements (animated arrows, zooming) permit us to cultivate cognitive skills. However, many different media could present a given attribute so there was no necessary 
correspondence between attributes and media. Media are mere vehicles for attributes so it is misleading to call them media attributes.

\section{Attributes and the Cultivation of Cognitive Skills}

The second expectation of the attribute approach was more realistic. While Mielke (1980) is correct that very few of the skill cultivating attributes have been found and validated, there is positive evidence for Salomons's (1979) claim that "the coding elements of a ... symbol system can be made to cultivate the mastery of specific mental skills by either activating or overtly supplanting the skills" (p. 216). (Much of the research buttressing this claim is presented in Salomon, 1979, and will not be reviewed in detail here.) The problem lies not in the fact that symbol systems can be made to cultivate skills but in whether these symbolic elements or attributes are exclusive or necessary to teaming. If the attributes identified to date are useful in instruction they are valuable. However, theory development depends on the discovery of basic or necessary processes of instruction and teaming. It is to this point, the third expectation of media attribute theories, that the discussion turns next.

\section{Attributes as Causal Factors in Learning}

There is recent evidence that attributes of symbol systems are occasionally sufficient but not necessary contributors to teaming. In science, sufficient conditions are those events which were adequate to produce some outcome in a past instance. There is no guarantee, however, that sufficient conditions will ever produce the outcome again because the variable that caused the outcome was merely correlated with the condition. For example, a lecture might be sufficient to produce the desired level of achievement in one instance but fail in another. Severing the optic nerve is sufficient to cause blindness but will not explain the cause of all cases of blindness. This issue is related to the problem of external validity. While it is often useful instructionally to know about sufficient conditions for producing desirable levels of achievement, our theories seek necessary conditions. Without necessary conditions we run the risk of failing to replicate achievement gains when we change the context, times, or student clients for instruction. Instructional theory (Shuell, 1980) .seeks- generalizations concerning the necessary instructional methods required to foster cognitive processes. To illustrate this point, the discussion turns next to research evidence for the skill-cultivating function of symbol system attributes.

\section{The Research Evidence}

In the zooming study mentioned earlier, students who had difficulty attending to cues in a visual field learned the skill by seeing it modeled in a film where they saw a camera "zoom" from a wide field to close-up shots of many different details (Salomon, 1974a). Here, an analysis of the task suggested that effective cue attending required an attention-directing strategy which began with a view of the entire stimulus and then narrowed the stimulus field until a single, identifiable cue

\section{p 452}

remained. For those students with low cue-attending skill (the requisite cognitive skill to perform the task) Salomon (1974b) reasoned that the required instructional method would be modeling. In this case, the construction of the model followed an analysis of the symbol systems, which allowed this particular method to be coded for delivery to the students. While the zooming treatment he used was available in many media (e.g., film, television, video disc) the students seemed to model the zooming and used it as a cognitive skill which allowed them to attend to cues.

However, in a partial replication of this study, Bovy (1983) found that a treatment which used an "irising" attribute to provide practice in cue-attending was as effective as Salomon's zooming in cultivating the skill during practice. Irising consisted of slowly enclosing cues in a circular, gradually enlarging, darkened border similar to the effect created by an iris which regulates the amount of light permitted through a camera lens. More important, however, was her finding that a treatment that merely isolated cues with a static close-up of successive details singled out by the zooming and irising was even more effective at cultivating cue-attending skill than either zooming or irising. It may be that only the efficient isolation of relevant cues is necessary for this task.

In a similar study, Blake (1977) taught chess moves to high or low visual ability undergraduates through a standard narration and (a) still pictures, (b) animated arrows with the pictures, or (c) a motion film from which the still pictures were taken. Therefore the recommendation is to exercise caution in future research on symbolic elements of media. While all three conditions worked for the higher ability students, low visualizers learned the chess moves equally well from the arrow and the motion treatments which were significantly better for them than the static pictures. Here, as in the Salomon (1974a) study, we presume that the modeled chess moves compensated for the low ability student's lack of spatial visualization. Unlike Salomon, Blake's subjects profited from two different 
operational definitions of the necessary model, animated arrows and moving chess pieces. Different stimulus arrangements resulted in similar performances but, we might expect, led to nominally different cognitive processes being modeled. The necessary process for learning chess moves, the visualizing of the entire move allowed each piece, could therefore be operationalized in any of various sufficient conditions for successful performance.

\section{Summary}

It seems reasonable to assume, therefore, that media are delivery vehicles for instruction and do not directly influence learning. However, certain elements of different media, such as animated motion or zooming, might serve as sufficient conditions to facilitate the learning of students who lack the skill being modeled. Symbolic elements such as zooming are not media (we can have a film or television program which does not contain zooming) but allow us to create sufficient conditions to teach required cognitive skills. The determination of necessary conditions is a fruitful approach when analyzing all instructional problems, and it is the foundation of all instructional theories. Once described, the necessary cognitive operation is a specification or recipe for an instructional method.

\section{p 453}

Of course, this point of view takes us a great distance from traditional conceptions of the role of media in instruction and learning. It suggests that systems of symbols that are correlated only with familiar media may sometimes serve as sufficient (but never necessary) conditions for learning from instruction. They accomplish this by providing operational vehicles for methods that reflect the cognitive processes necessary to successfully perform a given learning task. Generally, treatments such as zooming or animated arrows are but two of the many nominally different treatments which would result in the same performance. Just as some form of medium is required to deliver instruction, some form of a symbol system must be employed to construct a treatment. Similarly, as the medium does not influence learning, neither is the symbolic element chosen to construct the treatment the most direct influence on learning. We can employ a great variety of media and, possibly, a similar variety of symbol systems to achieve the same performance. However, we cannot vary the requirement that the method somehow model the crucial cognitive process required for the successful performance of the task. It is the critical features of the necessary cognitive process which underlie the construction of successful instructional methods and the development of instructional theory (Clark, 1982). These cognitive process features must be translated into a symbol system understandable to the learner and then delivered through a convenient medium. The cognitive feature in the chess study was the simulation of the beginning and ending points of the moves of the various chess pieces. In the cueattending studies by Salomon and Bovy, the cognitive features were probably the isolation of relevant cues. It is the external modeling of these features in a compatible symbol system that is necessary for learning. It is therefore not the medium or the symbol system that yields the required performance. When a chosen symbol system is shaped to represent the critical features of the task and other things are equal, learning will occur. When a medium delivers a symbol system containing this necessary arrangement of features, learning will occur also but will not be due to either the medium or the symbol system.

Of course there are instructional problems other than learning that may be influenced by media (e.g., costs, distribution, the adequacy of different vehicles to carry different symbol systems, equity of access to instruction). While space prevents a complete discussion of these more promising areas, what follows is a brief overview of studies that deal with research on our beliefs and attributions concerning media. It should be noted that these new questions differ from traditional media research

in that there are no media variables in the studies - only variables having to do with our attributions or beliefs about media. This is a subtle but important difference, as we shall see.

\section{Promising Research: Beliefs and Attributions About Media}

The fact that we learn (through education and experience) to prefer some media or to attribute varying levels of difficulty, entertainment value, or enjoyment to media might influence instructionally relevant outcomes. Several studies have fruitfully explored the attribution question. 
Perceived Learning Demands of Media and Learning Strategies

Presumably, differences in the qualities attributed to different media may influence learning-related behaviors of students. Ksobiech (1976) and Salomon (1981) have reported studies where student beliefs about the different demands placed on them by different media influenced their approach to learning tasks. Ksobiech (1976) told 60 undergraduates that televised and textual lessons were to be (a) evaluated, (b) entertainment, or (c) the subject of a test. The test group performed best on a subsequent test with the evaluation group scoring next best and the entertainment group demonstrating the poorest performance. Also in this study some subjects were allowed to push a button and receive more video or more narrative content (verbal information) about the lessons. The test subjects consistently chose more verbal-information presumably because they believed that it was a surer route to the factual information they needed to succeed at the test. Also, the subjects who believed that a test awaited them persisted longer than the other groups.

Salomon (1981) has recently suggested a model for conceptualizing these differences in persistence which result from different media attributions. His model suggests precise relationships between the perceived "difficulty" of different media, the self-efficacy of students, and the resulting effort they will invest in learning from a given medium. Again, it is the students' perception or attributions of the medium and their own abilities that -are thought to be causally connected to the effort they invest, not the medium per se. It is typical, Salomon reports, for students to attribute great difficulty to learning from computers but to think of television as "shallow" and "easy."

Enjoyment, Achievement, and Choice ofmedia

In related studies, Saracho (1982), Machula (1978-1979) and Clark (1982) reported studies where preferences and achievement from media were antagonistic. In a year-long study involving over 250 third- to sixth-grade students, Saracho (1982) found that those assigned to computer-assisted instruction in basic skills liked the computer less but learned more in the computer condition than from other media. Similarly, Machula (1978-1979) instructed 114 undergraduates via television, voice recording, and printed text. Students liked the television less but learned significantly more from it than from the voice recording, which they liked more.

Clark (1982) has reviewed similar studies and has suggested that by mistake students choose those media carrying methods that inadvertently result in less learning for them. Students incorrectly assess the extent to which the instructional method carried by the medium will allow them the most efficient use of their effort. Strong interactions with general abilities are often found in this research. Higher ability students seem to like methods and media that they perceive as more structured and directive because they think they will have to invest less effort to achieve success. However, these more structured methods prevent the higher ability student from employing their own considerable skills and yield less effort than t less directive methods and media. Lower ability students seem to typically like less structured and more discovery-oriented methods and media. They seem to want

\section{p 455}

to avoid investing the effort required by the more structured approaches to achieve the same disappointing results. These more unstructured approaches offer relative anonymity and the chance to invest less effort for the less able student who, on the other hand, actually needs the greater structure of the methods they like less. While medium and method are not the same experience, the methods conveyed by the media in studies such as these probably account for different levels of achievement while subject attributions about media influence their preferences.

\section{Attitudes Toward Computers}

Hess and Tenezakis (1973) explored the affective responses of predominantly Mexican-American, low SES seventh, eighth and ninth graders to remedial mathematics presented by computer or teacher. Among a number of interesting findings was an unanticipated attribution of more fairness to the computer than to the teacher. It seems that these subjects felt that the computer treated them more equitably (kept promises, did not make decisions based on their heritage) than some of the teachers. They consistently trusted the computer more but also found the computer to be less "flexible" and unresponsive to student desires to change the course or content of their instruction. Stimmel, Connor, McCaskill and Durrett (1981) found strong negative affect toward computers and computer instruction among a large group of preservice teachers. These same teacher trainees had similar reactions to mathematics and science teaching, and they may have associated computers with these disciplines.

\section{Conclusions}


One might reasonably wonder why media are still advocated for their ability to increase learning when research clearly indicates that such benefits are not forthcoming. Of course such conclusions are disseminated slowly and must compete with the advertising budgets of the multimillion dollar industry which has a vested interest in selling these machines for instruction. In many ways the problem is analogous to one that occurs in the pharmaceutical industry. There we find arguments concerning the relative effectiveness of different media (tablets, capsules, liquid suspensions) and different brand names carrying the same generic drug to users.

An equal contributor to this disparity between research and practice is the high expectation we have for technology of all kinds. Other machine-based technologies similar to the newer electronic media have revolutionized industry and we have had understandable hopes that they would also benefit instruction. And, there is the fact that many educators and researchers are reserved about the effectiveness of our system of formal education. As environments for learning, media seem to offer alternative and more effective features than those available from the conventional teacher in the conventional classroom. Tobias (1982) for example, has provided evidence that we can help overcome student anxiety by allowing anxious students the chance to replay a recording of a lesson. This quality of "reviewability" is commonly thought to distinguish some of the newer media from the conventional teacher's lecture. It is important to note however, that teachers are entirely capable of reviewing material for anxious students (and probably do so often). It is what the teacher does - the teaching - that influences learning. Most of the methods

\section{p 456}

carried by newer media can also be carried or performed by teachers. Dixon and Judd (1977), for example, compared teacher and computer use of "branching" rules in instruction and found no differences in student achievement attributable to these two "media."

The point is made, therefore, that all current reviews of media comparison studies suggest that we will not find learning differences that can be unambiguously attributed to any medium of instruction. It seems that existing research is vulnerable to rival hypotheses concerning the uncontrolled effects of instructional method and novelty.

More recent evidence questions the evidence for the media-based attempts to determine the components of effective instructional methods. These symbol system or media attribute theories. seem to be useful for instructional design but of limited utility in explicating the necessary conditions that must be met by effective methods. Future research should therefore focus on necessary characteristics of instructional methods and other variables (task, learner aptitude, and attributions), which are more fruitful sources for understanding achievement increases. Recent studies dealing with learner attributions and beliefs about the instructional and entertainment qualities of different media seem particularly attractive as research directions. There are no media variables in attribution research, however. Independent variables are concerned with learner beliefs, and outcome measures are typically some measure of learner persistence at a task. It seems reasonable to recommend, therefore, that researchers refrain from producing additional studies exploring the relationship between media and learning unless a novel theory is suggested.

\section{References}

Blake, T. Motion in instructional media: Some subject-display mode interactions. Perceptual and Motor Skills, 1977, 44, 975-985.

Bovy, R. A. Defining the psychologically active features of instructional treatments designed to facilitate cite attendance. Paper presented at the annual meeting of the American Educational Research Association, Montreal, April 1983.

Clark, R. E. Constructing a taxonomy of media attributes for research purposes. AV Communication Review, 1975, 23(2), 197-215.

Clark, R. E. Antagonism between achievement and enjoyment in ATI studies. The Educational Psychologist, 1982, $17(2), 92-101$.

Clark, R. E., \& Snow, R. E. Alternative designs for instructional technology research. AV Communication Review, 1975, 23(4), 373-394.

Cohen, J. Statistical power analysis for the behavioral sciences (Rev. ed.). New York: Academic Press, 1977.

Cohen, P., Ebling, B., \& Kulik, J. A meta-analysis of outcome studies of visual based instruction. Educational Communication and Technology Journal, 1981, 29(l), 26-36.

Dixon, P., \& Judd, W. A comparison of computer managed instruction and lecture mode for teaching basic statistics. Journal of Computer Based Instruction, 1977, 4(i), 22-25. 
Glaser, R. Components of a psychology of instruction: Towards a science of design. Review of Educational Research, 1976, 46(l), 1-24.

Glaser, R., \& Cooley, W. W. Instrumentation for teaching and instructional management. In R. Travers (Ed.), Second handbook of research on teaching. Chicago: Rand McNally College Publishing, 1973.

Glass, G. V. Primary, secondary and meta-analysis of research. Educational Researcher, 1976, 5(10), 3-8.

\section{p 457}

Hess, R., \& Tenezakis, M. The computer as a socializing agent: Some socio-affective outcomes of CAI. A V Communication Review, 1973, 21(3), 311-325.

Hovland, C., Lumsdaine, A. A., \& Sheffield, F. Experiments on mass communication. Princeton, N.J.: Princeton University Press, 1949.

Jamison, D., Suppes, P., \& Welles, S. The effectiveness of alternative instructional media: A survey. Review of Educational Research, 1974, 44, 1-68.

Keller, F. Good-bye teacher. Journal of applied Behavior Analysis, 1968, 1, 79-89.

Ksobiech, K. The importance of perceived task and type of presentation in student response to instructional television. Audio Visual Communication Review, 1976, 24(4), 401-41 1.

Kulik, C., Kulik, J., \& Cohen, P. Instructional technology and college teaching. Teaching Of Psychology, 1980, 7(4), 199-205.

Kulik, J., Bangert, R., \& Williams, G. Effects of computer-based teaching on secondary school students. Journal of educational Psychology, 1983, 75, 19-26.

Kulik, J., Kulik, C., \& Cohen, P. Research on audio-tutorial instruction: A meta-analysis of comparative studies. ' Research in Higher Education, 1979, 1](4), 321-34 1.

Levie, W. H., \& Dickie, K. The analysis and application of media. In R. Travers (Ed.), The second handbook of research on teaching. Chicago: Rand McNally, 1973.

Lumsdaine, A. Instruments and media of instruction. In N. Gage (Ed.), Handbook of research on teaching. Chicago: Rand McNally, 1963.

Machula, R. Media and affect: A comparison of videotape, audiotape and print. Journal of Educational Technology Systems, 1978-1979, 7(2), 167-185.

Mielke, K. Questioning the questions of ETV research. Educational Broadcasting Review, 1968,2,6-15.

Mielke, K. Commentary. Educational Communications and Technology Journal, 1980,28(i), 66-69.

Olson, D. On a theory of instruction: Why different forms of instruction result in similar knowledge. Interchange, 1972, 3(i), 9-24.

Olson, D., \& Bruner, J. Learning through experience and learning through media. In D.

Olson (Ed.), Media and symbols.- The forms of expression, communication, and education (73rd Yearbook of the NSSE). Chicago: University of Chicago Press, 1974.

Reiser, R. \& Gagne, R. Characteristics of media selection models. Review of Educational Research, 1982, 52(4), 499-512.

Rosenshine, B. Teacher behaviors and student achievement. London: National Foundation for Educational Research in England and Wales, 1971.

Salomon, G. Internalization of filmic schematic operations in interaction with learners' aptitudes. Journal of educational Psychology, 1974, 66, 499-51 1. (a)

Salomon, G. What is learned and how it is taught: The interaction between media, message, task and learner. In D. Olson (Ed.), Media and symbols.- The forms of expression, communication, and education (73rd Yearbook of the NSSE). Chicago: University of Chicago Press, 1974. (b)

Salomon, G. Interaction of media, cognition and learning. San Francisco!. Jossey Bass, 1979-. Salomon, G. Communication and education. Beverly Hills, Calif.: Sage, 1981.

Salomon, G. \& Clark, R. E. Reexamining the methodology of research on media and technology in education. Review of educational Research, 1977, 47, 99-120.

Saracho, 0. N. The effect of a computer assisted instruction program on basic skills achievement and attitude toward instruction of Spanish speaking migrant children. American Educational Research Journal, 1982, 19(2), 201219.

Schramm, W. Big media little media. Beverly Hills, Calif.: Sage, 1977.

Shuell, T. J. Learning theory, instructional theory and adaption. In R. E. Snow, P. Federico, \& W. Montigue (Eds.), Aptitude, learning and instruction (Vol. 2). Hillsdale, N.J.: Lawrence Erlbaum, 1980. 
Stimmel, T., Connor, J., McCaskill, E., \& Durrett, H. J. Teacher resistance to computer assisted instruction. Behavior Research Methods and Instrumentation, 1981, 13(2), 128-130.

\section{p 458}

Thorndike, E. L. Education. New York: Macmillan, 1912.

Tobias, S. When do instructional methods make a difference? Educational Researcher, 1982, 1](4), 4-9.

\section{AUTHOR}

RICHARD E. CLARK, Professor of Educational Psychology and Technology, University of Southern California, WPH 801 P. 0. Box 77963, Los Angeles, CA 90007. Specialization: Instructional theory. 